\title{
Interactive comment on "Early results and Validation of SAGE III-ISS Ozone Profile Measurements from Onboard the International Space Station” by Michael P. McCormick et al.
}

\section{Anonymous Referee \#2}

Received and published: 8 November 2019

\section{Short resume}

This paper discusses the first validation activities on SAGE III-ISS ozone occultation measurements. These data continue and add value to the long-term SAGE time series. As a reference for the validation, two stations equipped with ground-based lidar and ozonesonde are selected. In addition, a comparison is performed with respect to ACEFTS observations. The early results of the validation are presented in a structured way with a clear description of the procedure followed for the comparison. The description of the results could be improved. 
This paper fits the scope of AMT, and it is logically written. From my side, I have some comments on specific aspects and technical corrections.

\section{Specific comments}

Interactive

comment

1. Usage of SAGE III-ISS profiles

In section 2.1, the authors introduce the four retrieved ozone products but I could not find in the text which one is then used for the data analysis. Could you please provide some more details about this point and, if available, some characterization of the retrieved profiles? For example, the vertical resolution of ACE-FTS ozone profiles is mentioned in Section 2.3 and a similar information for SAGE IIIISS would be interesting as well. Could you add a reference about the retrieval algorithm, if available, and the url of the Data Products User's Guide at line 70?

2. Why were precisely Lauder and Hohenpeissenberg stations selected instead of several stations at northern and southern mid-latitudes? Is there any specific reason?

3. If I understood it correctly, the authors have always used a linear interpolation to compare higher resolution profiles from correlative data sets with SAGE III-ISS observations. Did you think about the use of averaging kernels from SAGE III-ISS retrievals, especially when comparing ozonesonde data? Their use may improve the comparison in the lower stratosphere and reduce oscillations in the relative differences (e.g. Fig. 11).

4. Collocation criteria

I found the collocation criteria used for ozonesonde/lidar particularly loose in terms of time coincidence (a window of 96 hours around the observations, right?) and pretty strict in the spatial domain: the longitude requirement of $5^{\circ}$ could be in 
my mind slightly relaxed to find more collocations, as chosen for ACE-FTS. Could you justify this choice of criteria? A general better motivation of the collocation AMTD criteria would be appreciated.

5. I found the description of the second part of Sect. 2.4 clear and well structured. On the contrary, I found confusing the quantities shown in Figs. 3 and following. In the left panel, do the error bars correspond to the standard deviation, like the quantity in Eq. (3), or is it the average of the precision of the considered profiles (which, however, in Fig. 2 was almost not visible)? In the right panel, do you plot the percentage difference between the averaged profiles from the two instruments (as I understood from the caption) or $\Delta(z)$ from Eq.(1)? Is the standard error equal to the SEM of Eq. (4)?

6. The use of $r e f_{i}(z)$ as described in Eq. (2) is to me well justifiable for the comparison with ACE-FTS profiles, being also satellite observations, which need to be validated against ground-based data. On the contrary, when comparing SAGE III-ISS with ozonesonde and lidar profiles I would use the reference profile itself in the denominator instead of Eq. (2). This is also done by Randall et al. (2003), which is also cited by the authors.

7. I am confused with the collocations between SAGE III-ISS and ACE-FTS shown in Fig. 7b. From the beginning it is to me not clear that these are the only available collocations. On the contrary, it seems that the plot refers to the northern hemisphere only for graphical purposes, as Fig. 7 caption says 'only northern hemisphere data shown'. Then at line 185: 'Coincident events are MOSTLY located at mid-latitudes and high-latitudes in the northern hemisphere.... On the next page, I find the clear statement at line 203: '...coincident events [...] could only be found in the northern hemisphere under the criteria.... Could you please clarify this and, in case, move this last sentence up to line $185 ?$

Interactive comment

Printer-friendly version

Discussion paper

8. I find panels (a) and (b) of Fig. 10 not so quantitative interesting, as it is difficult 
to estimate the differences between the two plots by eye. I would rather expand panel (c) of this picture and leave it alone so that the differences as a function of AMTD latitude and altitude are better visible.

9. At line 237, cirrus clouds are indicated as responsible for discrepancies between SAGE III-ISS and ACE-FTS, how do you filter out clouds from measurements?

Interactive comment

10. For the lunar occultation, why don't you show the average of the 7 available coincidences with ACE-FTS instead of one example only (Fig. 12)?

11. The layout of the figures

I think that the layout and visibility of some pictures would strongly improve by reducing the font of the writings in the plots. I consider Fig. 2 as an example but this is applicable also to Figs. 3, 4, 5, 6, 11 and 12. On the left panel, could you please reduce the text information in the plot area and their font size? In this way, the $x$-axis could be zoomed to the range $0-6 * 10^{12}$, and the profiles would be more visible. For example, the information about latitude and longitude of the station is constant and is already mentioned in Sect. 2.2. I also find the x-axis of the right panel too wide: it would be more interesting to better see the differences in the $15-40 \mathrm{~km}$ range. I suggest expanding the range to [-50, 50]. Indeed, the large relative differences in the lowermost and uppermost layers are anyway cut in some plots, even keeping the $[-100,100]$ range. In addition, the text on the right panel is, in my opinion, redundant, as always mentioned in the text as well. By removing this, you avoid the overlap of some writing with the profiles, as in Fig. 4. Similar suggestions, though not so critical as before, I would recommend for Figs. 8 and 9. Expanding the x-axis would ease the visibility of the profiles and of the differences.

12. Verb Tenses

I noted that in different parts of the paper inconsistent verb tenses are used.

For example, in Sect. 2.4, after using the past tense to describe the collocation 
procedure, the future/present tense is introduced. Another example: in Sect. 3.2, the whole description of Figs. 5 and 6 is in the past, whereas Fig. 4 is described AMTD in the present.

\section{Technical corrections}

Interactive comment

P1, I28: I would also mention the impact of the climate change on the ozone layer (e.g. BDC modification, stratospheric cooling), not only the impact of ozone changes in the climate.

P1, 141: 'record of observation' $\rightarrow$ 'record of observations'

P2, 146: change to 'SAGE III-ISS ozone profiles with observations made by ...'

P4, 1124-126: I would avoid repeating the description of the single terms, which are already explained after Eq. (1).

P4, I131: As the last paragraph title includes the lunar adjective, I would also specify here 'SAGE III-ISS solar' measurements.

P4, caption Fig. 2: check the spelling of Hohenpeissenberg

P5, caption Fig. 3: check the spelling of Hohenpeissenberg

P5, I145: I would delete one ozone in the expression 'Hohenpeissenberg ozone lidar ozone data'

P6, 1158: Same as for the title of Sect. 3.1

P6, I164: please add a reference to the statement about the pump efficiency.

P7, top: something is wrong with the font of the section title.

P7, 1187: wrong parenthesis, ] $\rightarrow$ )

P7, I189 and 1211: profile $\rightarrow$ profiles

P7, I191-206: I think it is not necessary to repeat the legend of the figure in the text.

Printer-friendly version

P8, 1207: describe $\rightarrow$ described

Discussion paper

P8, I215: 'Figure 9 a, c, e, g' replace with 'Figures 9 b, d, f, h'

P8, 1216: result $\rightarrow$ shows 
P11, I252-253: I would delete the sentence 'The time difference for this ... in distance'. P11, I268: what does it mean 'the impact of ozone on variation studies'?

AMTD

P11, I272: were obtain $\rightarrow$ were obtained.

The term 'northern hemisphere' is written sometimes capitalized and other times small. Please check the correct use of Fig. and Figs., e.g lines 210, 214, 233.

Interactive comment

Interactive comment on Atmos. Meas. Tech. Discuss., doi:10.5194/amt-2019-353, 2019. 